\title{
Simulação realística como ferramenta de ensino na saúde: uma revisão integrativa
}

\author{
Realistic simulation as a teaching tool in health: an integrative review \\ Simulación realista como herramienta de enseñanza en la salud: una \\ revisión integradora
}

\begin{abstract}
Marcelo Tsuyoshi Yamane ${ }^{1}$, Vinicius Klettenberg Machado², Karyna
Turra Osternack ${ }^{3}$, Rosiane Guetter Mello ${ }^{4}$
\end{abstract}

\footnotetext{
${ }^{1}$ Acadêmico do Curso de Medicina da Faculdades Pequeno Príncipe - FPP Curitiba - Paraná

${ }^{2}$ Acadêmico do Curso de Medicina da Faculdades Pequeno Príncipe - FPP Curitiba - Paraná

${ }^{3}$ Mestre em Biotecnologia Aplicada à Saúde da Criança e do Adolescente pela Faculdades Pequeno Príncipe - FPP Curitiba - Paraná

${ }^{4}$ Doutora em Ciências (Bioquímica) pela UFPR. Docente do Programa de Mestrado em Ensino nas Ciências da Saúde da Faculdades Pequeno Príncipe Curitiba - Paraná
}

\section{RESUMO}

\begin{abstract}
A forma de ensino na saúde mudou em todo o mundo, com a implementação da simulação realística, que adquiriu um papel importante na educação, porque é uma forma ativa de aprendizado. O estudo objetivou pesquisar publicações brasileiras sobre a utilização dessa metodologia de ensino-aprendizagem nas escolas de saúde, analisando os cursos e áreas que mais utilizam a técnica, limitações, desafios e benefícios alcançados. Trata-se de uma revisão integrativa da literatura. Totalizaram-se 25 publicações, selecionadas nas bases de dados BVS e SciELO utilizando os descritores "simulação realística" e "ensino baseado em simulação".
\end{abstract}

Autor de Correspondência:

*Marcelo Tsuyoshi Yamane - E-mail: marcelo-tsuyoshi@hotmail.com 
Constatou-se que a simulação é metodologia indispensável no ensino em saúde, porque promove o protagonismo do estudante no processo ensino-aprendizagem. Concluiu-se que a simulação pode ser utilizada no desenvolvimento de competências essenciais do profissional de saúde. No entanto, existem limitações na sua implementação. Também são escassos os estudos além das graduações de medicina e enfermagem e na atenção primária.

Palavras-Chave: Treinamento por Simulação. Competência Profissional. Instituições de Ensino.

\begin{abstract}
Health education has changed all over the world, with the implementation of realistic simulation, which has acquired an important role in education, because it is an active form of learning. The study aimed to collect Brazilian publications about the implementation of this teaching-learning methodology in health schools, analyzing the courses and areas that use the technique the most, limitations, challenges and benefits achieved. This is an integrative review of the literature. Twenty-five publications were selected in the BVS and SciELO databases using the descriptors "realistic simulation" and "simulation-based teaching". It was observed that simulation is an indispensable methodology in health teaching, because it promotes the protagonism of the student in the teaching-learning process. It was concluded that simulation can be used in the development of essential competences of the health professional. However, there are limitations in its implementation. Studies are also scarce beyond medical and nursing undergraduation courses, and in primary care.
\end{abstract}

Keywords: Simulation Training. Professional Competence. Health Area Education Centers.

\title{
RESUMEN
}

La forma de la enseñanza en la salud ha cambiado en todo el mundo, con la implementación de la simulación realista, que ha adquirido un papel importante en la educación, porque es una forma activa de aprendizaje. El estudio objetivó levantar publicaciones brasileñas sobre la implementación de esa metodología de enseñanza aprendizaje en las escuelas de salud, analizando los cursos y áreas que más utilizan la técnica, limitaciones, desafíos y beneficios alcanzados. Se trata de una revisión integradora. Se totalizaron 25 publicaciones, seleccionadas en las bases de datos BVS y SciELO utilizando los descriptores "simulación realista” y "enseñanza basada en simulación”. Se constató que la simulación es metodología indispensable en la enseñanza en salud, porque promueve el protagonismo del estudiante en el proceso enseñanza aprendizaje. Se concluyó que la simulación puede ser utilizada en el desarrollo de competencias esenciales del profesional de salud. Sin embargo, existen limitaciones en su implementación. También son escasos los estudios más allá de las graduaciones de medicina y enfermería y en la atención primaria.

Palabras Clave: Entrenamiento Simulado. Competencia Profesional. Centros Educacionales de Áreas de Salud 


\section{INTRODUÇÃO}

Nas últimas décadas a forma do ensino na área de saúde mudou em todo o mundo. O ensino tradicional, em que o professor é o transmissor do conhecimento, já não é mais compatível com as necessidades da área da saúde. É por isso que as grandes escolas tentam desenvolver e implementar um aprendizado construído no exercício do aprender fazendo, nas relações constantes entre teoria e a prática, no pensamento criativo e na aprendizagem significativa. Pensando nisso, escolas dos EUA há algum tempo implantaram em seu currículo as simulações realísticas no ensino das escolas médicas e um pouco mais posteriormente, universidades do Reino Unido também implementaram e obtiveram bons resultados, como vistas pelo professor Ronald Harden da University of Dundee. É dessa maneira que a simulação adquiriu um papel importante na educação médica, já que é uma forma ativa de aprendizado, em que o aluno se vê no cenário que encontrará na prática, sem, no entanto, correr os riscos que encontrará no ambiente real ${ }^{1}$.

Os números relacionados a impactos negativos dos profissionais médicos no exercício da profissão sob os pacientes têm se tornado alarmantes. Nos EUA erros médicos preveníveis causam mais de 400.000 mortes e são responsáveis por mais de 3,5 milhões de iatrogenias, levantando a possibilidade de que a cultura de ensino da medicina não seja mais adequada ${ }^{2}$. Nesse sentido, como método alternativo, a simulação tem mostrado resultados impressionantes, tanto na redução dos números de erros médicos, quanto na melhoria da associação de conteúdos ${ }^{3}$.

A primeira experiência próxima à simulação na prática clínica se deu no século XVIII, em Paris, quando os Grégorie, pai e filho, desenvolveram um manequim de pelve feminina para o ensinamento de técnicas obstétricas. Esta ação, por si, resultou em reduções nas taxas de óbito materno e infantil. Já o primeiro modelo de simulação surgiu na área aeroespacial, com a invenção da "Blue Box” em 1929, sendo esta capaz de reproduzir os movimentos e sensações causados no voo ${ }^{2}$.

A indústria aeroespacial a partir de então tem grandes avanços tecnológicos na área de simulação de situações de voo, e com isso tem sido possível uma redução de 50\% dos acidentes aeroespaciais. Da mesma forma, no mesmo século, em 1960 Ausmund Laerdal desenvolveu um manequim de simulação para aprendizado da ventilação boca a boca, chamado Resusci-Anne, possibilitando o desenvolvimento e treinamento de técnicas de ressuscitação em modelos ${ }^{2}$.

A partir da simulação, garante-se, então, a experiência de um evento tal qual o real, em um ambiente seguro ${ }^{4}$. Na perspectiva do estudante, a simulação permite simular condições ideais e aplicar os conhecimentos com sua magnitude e plenitude, com a possibilidade de refletir a respeito de seus próprios erros na simulação. $\mathrm{Na}$ perspectiva do paciente é útil no desenvolvimento de competências e habilidades no estudante para sua atuação direta com o paciente e garante uma prática permissiva de erros que não os causam danos ${ }^{3}$. A simulação garante, pois, um ambiente seguro e controlado, com possibilidade de variações de conteúdo e de níveis de dificuldades, prevenindo potenciais riscos aos pacientes, garantindo assim o princípio essencial de "primum non nocere", alinhado com as recomendações internacionais de reestruturação da educação médica ${ }^{2,4}$.

A teoria de aprendizado do adulto sugere que existem grandes diferenças entre a pedagogia e a andragogia. É provado, por essa teoria, que o modelo centrado no estudante tem demonstrado resultados consistentes de aprendizado, diferentemente das aulas listadas e capítulos prefixados do modelo tradicional $^{2}$. Portanto, sendo a simulação uma metodologia centrada no educando, e facilitadora na redução dos índices de morbimortalidade, pode ser utilizada como ferramenta única no processo 
de ensino-aprendizagem e como uma ferramenta complementar de outros métodos, possibilitando uma fixação mais concreta do conteúdo ${ }^{4}$.

A simulação é versátil, sendo possível seu uso nas mais diversas áreas da medicina, tanto clínicas quanto cirúrgicas. A sua construção se dá apoiada no desenvolvimento de três peças-chave sumarizadas em escopo (o conteúdo que será abrangido na simulação), a modalidade (se a simulação se dará por meio de jogos interativos, por meio de manequins ou atores treinados, por exemplo) e ambiente (os materiais e meios utilizados para aproximar a simulação das situações reais). Essa construção é essencial e não exige altas tecnologias e manequins de alta resposta. O educador deve delinear à atividade de simulação considerando a especificidade, mensurabilidade e abrangência da ação, com foco nos resultados a serem atingidos pela atividade e nos elementos requeridos pela atividade ${ }^{4}$.

Uma parte crítica na construção é o debriefing, realizado no final representando uma oportunidade ímpar para o reforço dos assuntos tratados na simulação, guiando um processo de reflexão ${ }^{2}$. Pode ser lançado mão de vídeos gravados durante as simulações para relembrar as ações, utilizá-los em outros momentos educativos ou ainda no mesmo momento de debriefing 4 .

Sendo assim, a simulação é uma ferramenta de ensino-aprendizagem que corrobora na obtenção e fixação de conteúdos, garantindo a segurança do paciente e reduzindo a morbimortalidade relacionada a iatrogenias. O estudo teve como objetivo pesquisar publicações brasileiras sobre a implementação das simulações realísticas nas escolas de saúde brasileiras nos últimos dez anos, analisando os cursos e áreas que mais utilizam a técnica, as limitações e desafios dessa metodologia de ensino-aprendizagem e os benefícios alcançados.

\section{MÉTODO}

Trata-se de um estudo de revisão integrativa, que objetiva reunir e sintetizar resultados de pesquisas sobre um tema ou questão, de maneira sistemática e ordenada, contribuindo para o aprofundamento do conhecimento do tema investigado e direcionando a prática fundamentada em conhecimento científico. Além disso, possibilita mostrar lacunas no conhecimento e explicitar assuntos pouco estudados ${ }^{5}$

O processo de criação de uma revisão integrativa encontra-se bem definido na literatura. Para a elaboração desse tipo de pesquisa é preciso realizar seis etapas, similares aos encontrados na pesquisa convencional: 1 1 - identificação do tema e seleção da hipótese ou questão de pesquisa para a elaboração da revisão integrativa; $2^{\underline{a}}$ - estabelecimento de critérios para inclusão e exclusão de estudos/ amostragem ou busca na literatura; $3^{\mathbf{a}}$ - definição das informações a serem extraídas dos estudos selecionados/ categorização dos estudos; $4^{\text {a }}$ - avaliação dos estudos incluídos na revisão integrativa; $5^{\mathbf{a}}$ - interpretação dos resultados; 6 - apresentação da revisão/síntese do conhecimento5.

Dessa maneira, realizou-se uma busca bibliográfica para selecionar publicações sobre o tema proposto. Os artigos foram consultados nas bases de dados BVS e SciELO. A pesquisa foi feita por meio dos descritores "simulação realística" e "ensino baseado em simulação". Foram utilizados artigos escritos em português publicados no período de 2010 a 2019 e incluídos artigos originais, pesquisas quantitativas e qualitativas, estudos retrospectivos, artigos de revisão sobre o tema e estudos de casos.

Na BVS, aplicando-se os critérios de busca foram encontradas 460 publicações. Os seguintes filtros foram colocados como critérios de inclusão: texto completo disponível, publicação brasileira, artigo em português e anos de publicação entre 2010 e 2019, restando 49 publicações. Realizando a leitura do título e resumo dos artigos, foram excluídos 31, 
Figura 1 - Fluxograma das etapas de busca

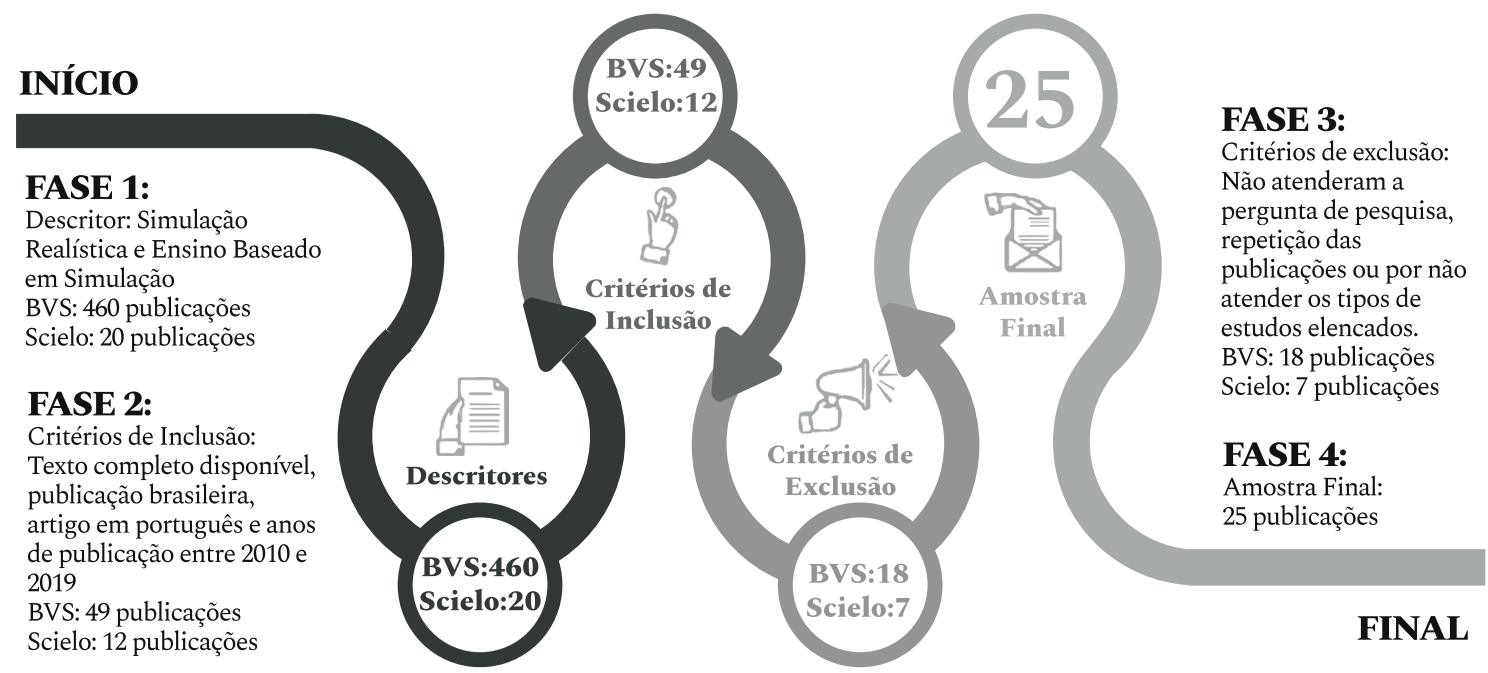

em razão de não atenderem a pergunta de pesquisa, pela repetição das publicações ou por não atender os tipos de estudo supracitados. Na SciELO, foram encontradas 20 publicações. Aplicando-se os mesmos critérios de seleção, 13 artigos foram excluídos. O fluxograma com as etapas de busca nas bases de dados está mostrado na Figura 1.

Ao final da seleção, o estudo totalizou 25 artigos que foram categorizados e analisados de acordo com título, autores, revista, ano de publicação, área da publicação, objetivos, método e resultados. Para a realização da análise das publicações foi realizada a leitura na íntegra dos trabalhos. Depois de organizados os dados, a partir da sumarização e síntese dos conhecimentos acumulados e interrelação dos resultados dos estudos anteriores, foi realizada análise de forma crítica, para produzir novo conhecimento integrado.

\section{RESULTADOS}

A síntese do levantamento será apresentada na tabela 1 , onde foram selecionadas as informações relevantes das publicações selecionadas, como: título, autores, revista, ano e área de publicação.

Tabela 1 - Artigos selecionados para a revisão integrativa de literatura

\begin{tabular}{llllll}
\hline Título & Autores & Revista & Ano & Área \\
\hline A Percepção de estudantes da & Costa et al. & $\begin{array}{c}\text { Revista } \\
\text { graduação em Enfermagem sobre a } \\
\text { simulação realística }\end{array}$ & & 2017 & Enfermagem \\
\hline
\end{tabular}




\begin{tabular}{|c|c|c|c|c|c|}
\hline & Título & Autores & Revista & Ano & Área \\
\hline B & $\begin{array}{l}\text { A simulação realística como } \\
\text { ferramenta educacional para } \\
\text { estudantes de Medicina }\end{array}$ & Brandão et al. & $\begin{array}{l}\text { Scientia } \\
\text { Medica }\end{array}$ & 2014 & Medicina \\
\hline C & $\begin{array}{l}\text { Vivência prática de simulação } \\
\text { realística no cuidado ao paciente } \\
\text { crítico: relato de experiência }\end{array}$ & Magro et al. & $\begin{array}{c}\text { Revista } \\
\text { Baiana de } \\
\text { Enfermagem }\end{array}$ & 2012 & Enfermagem \\
\hline D & $\begin{array}{l}\text { Ensino baseado em simulação na } \\
\text { formação continuada de médicos: } \\
\text { análise das percepções de alunos e } \\
\text { professores de um hospital do Rio de } \\
\text { Janeiro }\end{array}$ & $\begin{array}{l}\text { Dourado e } \\
\text { Giannella }\end{array}$ & $\begin{array}{c}\text { Revista } \\
\text { Brasileira } \\
\text { de Educação } \\
\text { Médica }\end{array}$ & 2014 & Medicina \\
\hline E & $\begin{array}{l}\text { Simulação de alta fidelidade realística } \\
\text { para o ensino de emergências } \\
\text { médicas na prática odontológica: } \\
\text { relato de experiência }\end{array}$ & Motta et al. & $\begin{array}{l}\text { Revista da } \\
\text { ABENO }\end{array}$ & 2018 & Odontologia \\
\hline $\mathrm{F}$ & $\begin{array}{l}\text { Ensino do brinquedo terapêutico na } \\
\text { graduação em enfermagem: Ações e } \\
\text { estratégias didáticas utilizadas por } \\
\text { professores }\end{array}$ & Maia et al. & $\begin{array}{c}\text { Texto \& } \\
\text { Contexto } \\
\text { Enfermagem }\end{array}$ & 2019 & Enfermagem \\
\hline G & $\begin{array}{l}\text { O uso da simulação em Enfermagem } \\
\text { no curso técnico de Enfermagem }\end{array}$ & Sanino & $\begin{array}{l}\text { Journal } \\
\text { Of Health } \\
\text { Informatics }\end{array}$ & 2012 & Enfermagem \\
\hline $\mathrm{H}$ & $\begin{array}{l}\text { Atitudes de estudantes iniciantes da } \\
\text { graduação em enfermagem diante do } \\
\text { paciente alcoolista }\end{array}$ & Rodrigues e Souza & $\begin{array}{l}\text { Revista } \\
\text { Eletrônica } \\
\text { Saúde Mental } \\
\text { Álcool e } \\
\text { Drogas }\end{array}$ & 2018 & Enfermagem \\
\hline
\end{tabular}

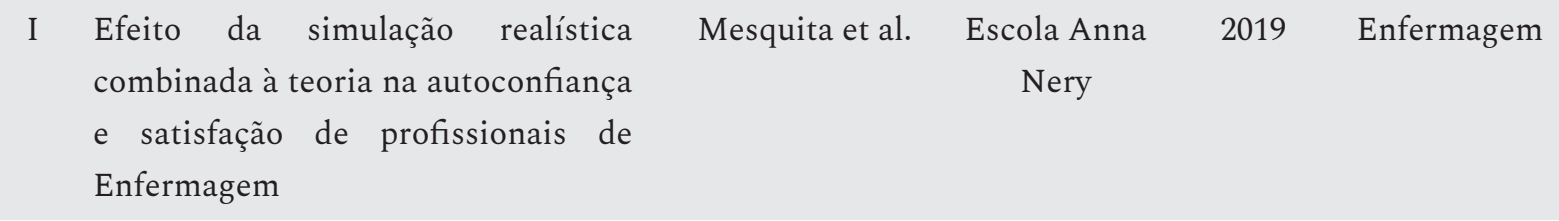




\begin{tabular}{|c|c|c|c|c|c|}
\hline & Título & Autores & Revista & Ano & Área \\
\hline $\mathrm{J}$ & $\begin{array}{l}\text { Simulação realística: método } \\
\text { de melhoria de conhecimento e } \\
\text { autoconfiança de estudantes de } \\
\text { enfermagem na administração de } \\
\text { medicamento }\end{array}$ & $\begin{array}{l}\text { Nascimento e } \\
\text { Magro }\end{array}$ & $\begin{array}{c}\text { Revista } \\
\text { Mineira de } \\
\text { Enfermagem }\end{array}$ & 2018 & Enfermagem \\
\hline K & $\begin{array}{l}\text { Impacto da metodologia de } \\
\text { simulação realística na graduação de } \\
\text { enfermagem }\end{array}$ & Rohrs et al. & $\begin{array}{l}\text { Revista de } \\
\text { Enfermagem } \\
\text { UFPE }\end{array}$ & 2017 & Enfermagem \\
\hline $\mathrm{L}$ & $\begin{array}{l}\text { Laboratório de enfermagem: } \\
\text { estratégias criativas de simulações } \\
\text { como procedimento pedagógico }\end{array}$ & Figueiredo & $\begin{array}{l}\text { Revista de } \\
\text { Enfermagem } \\
\text { UFSM }\end{array}$ & 2014 & Enfermagem \\
\hline M & $\begin{array}{l}\text { E-baby integridade da pele: inovação } \\
\text { tecnológica no ensino de } \\
\text { enfermagem neonatal baseado em } \\
\text { evidências }\end{array}$ & Aredes et al. & $\begin{array}{c}\text { Escola Anna } \\
\text { Nery }\end{array}$ & 2018 & Enfermagem \\
\hline $\mathrm{N}$ & Oftalmologia e realidade virtual & Carvalho & $\begin{array}{l}\text { Ver. } \\
\text { Brasileira de } \\
\text { Oftalmologia }\end{array}$ & 2012 & Medicina \\
\hline $\mathrm{O}$ & $\begin{array}{l}\text { Modelagem conceitual de simulador } \\
\text { virtual para treinamento em anestesia } \\
\text { regional }\end{array}$ & Costa et al. & $\begin{array}{l}\text { Journal } \\
\text { of Health } \\
\text { Informatics }\end{array}$ & 2016 & Medicina \\
\hline $\mathrm{P}$ & $\begin{array}{l}\text { Conhecimento teórico de estudantes } \\
\text { de enfermagem sobre o cateterismo } \\
\text { vesical de demora }\end{array}$ & Lopes et al. & $\begin{array}{l}\text { Revista de } \\
\text { Enfermagem } \\
\text { do Centro- } \\
\text { Oeste } \\
\text { Mineiro }\end{array}$ & 2018 & Enfermagem \\
\hline Q & $\begin{array}{l}\text { Modelo de avaliação de progressão } \\
\text { de competência e Habilidades em } \\
\text { endosuturas por meio de treinamento } \\
\text { em laboratório de habilidades } \\
\text { cirúrgicas }\end{array}$ & Moura-Junior et al. & $\begin{array}{l}\text { Arquivos } \\
\text { Brasileiros de } \\
\text { Cirurgia } \\
\text { Digestiva }\end{array}$ & 2017 & Medicina \\
\hline
\end{tabular}




\begin{tabular}{|c|c|c|c|c|c|}
\hline & Título & Autores & Revista & Ano & Área \\
\hline $\mathrm{R}$ & $\begin{array}{l}\text { Aula simulada no ensino de ações de } \\
\text { Enfermagem na intubação }\end{array}$ & Alves et al. & $\begin{array}{l}\text { Revista de } \\
\text { Enfermagem } \\
\text { UFPE }\end{array}$ & 2018 & Enfermagem \\
\hline$S$ & $\begin{array}{l}\text { Simulação realística como método de } \\
\text { ensino no aprendizado de estudantes } \\
\text { da área da saúde }\end{array}$ & Ferreira et al. & $\begin{array}{l}\text { Revista de } \\
\text { Enfermagem } \\
\text { do Centro- } \\
\text { Oeste } \\
\text { Mineiro }\end{array}$ & 2018 & $\begin{array}{c}\text { Enfermagem } \\
\text { e Medicina }\end{array}$ \\
\hline $\mathrm{T}$ & $\begin{array}{l}\text { Opinião dos estudantes de } \\
\text { Enfermagem sobre a simulação } \\
\text { realística e o estágio curricular em } \\
\text { cenário hospitalar }\end{array}$ & Valadares e Magro & $\begin{array}{c}\text { Acta } \\
\text { Paulista de } \\
\text { Enfermagem }\end{array}$ & 2014 & Enfermagem \\
\hline $\mathrm{U}$ & $\begin{array}{l}\text { Simulação in situ, uma metodologia } \\
\text { de treinamento multidisciplinar } \\
\text { para identificar oportunidades de } \\
\text { melhoria na segurança do paciente } \\
\text { em uma unidade de alto risco }\end{array}$ & Kaneko et al. & $\begin{array}{c}\text { Revista } \\
\text { Brasileira } \\
\text { de Educação } \\
\text { Médica }\end{array}$ & 2015 & $\begin{array}{c}\text { Enfermagem } \\
\text { e Medicina }\end{array}$ \\
\hline V & $\begin{array}{l}\text { Construção e desenvolvimento de } \\
\text { cenários de simulação realística } \\
\text { sobre a administração segura de } \\
\text { medicamentos }\end{array}$ & Cogo et al. & $\begin{array}{c}\text { Revista } \\
\text { Gaúcha } \\
\text { de } \\
\text { Enfermagem }\end{array}$ & 2019 & $\begin{array}{l}\text { Enfermagem } \\
\text { e Farmácia }\end{array}$ \\
\hline W & $\begin{array}{l}\text { Exame clínico objetivamente } \\
\text { estruturado no ensino de suporte } \\
\text { básico de vida }\end{array}$ & Oliveira e Silva & $\begin{array}{l}\text { Revista de } \\
\text { Enfermagem } \\
\text { UFPE }\end{array}$ & 2018 & Enfermagem \\
\hline $\mathrm{X}$ & $\begin{array}{l}\text { Simulação realística como estratégia } \\
\text { de ensino para o curso de graduação } \\
\text { em enfermagem: revisão integrativa }\end{array}$ & Barreto et al. & $\begin{array}{c}\text { Revista } \\
\text { Baiana de } \\
\text { Enfermagem }\end{array}$ & 2014 & Enfermagem \\
\hline Y & $\begin{array}{l}\text { O uso da simulação no contexto da } \\
\text { educação e formação em saúde e } \\
\text { Enfermagem: uma reflexão } \\
\text { acadêmica }\end{array}$ & Costa et al. & $\begin{array}{l}\text { Revista } \\
\text { Espaço para } \\
\text { a Saúde }\end{array}$ & 2015 & Enfermagem \\
\hline
\end{tabular}


A tabela 2 apresenta os objetivos, métodos e resultados dos artigos analisados, com o propósito de aprofundar a análise dos temas apresentados nas publicações componentes do estudo.

Tabela 2 - Conteúdo dos estudos

\section{Objetivos}

Métodos

Estudo descritivo A simulação foi percebida como uma

com abordagem técnica que permite uma vivência quanti- prévia da prática, permitindo aos qualitativa

\section{Resultados}

estudantes refletir sobre os seus
A Identificar a percepção de estudantes da graduação em Enfermagem sobre a simulação realística enquanto estratégia de ensino e aprendizagem. futuros contextos de trabalho. Além disso, permite relacionar a teoria e a prática, ajudando na aprendizagem dos conteúdos. Por ser uma estratégia dinâmica e ativa, a simulação promove integração e o desejo de expansão e outras vivências a partir de cenários simulados em outras disciplinas da graduação em Enfermagem.
B Realizar revisão de literatura sobre a utilização da simulação realística, enfatizando a graduação médica e seus aspectos mais relevantes e atuais.

\section{Revisão Integrativa Os artigos estudados relatam a importância e auxílio da tecnologia em agregar melhorias ao ensino médico. A segurança do paciente foi descrita como fator decisivo na implementação da simulação realística nas instituições de ensino mundiais. As habilidades em comunicação, liderança, tomada de decisão, trabalho em equipe e relacionamento médico/ paciente aparecem nesta revisão como o maior benefício de informação aos estudantes.}

C Descrever a experiência de um grupo de alunos na implementação da simulação realística.

\section{Relato de} experiência
O cenário de simulação possibilita ao aluno vivenciar, em tempo real, situações frequentes na prática do cuidado, em que a consolidação 


\section{Objetivos}

Métodos

\section{Resultados}

do conhecimento e experiências ocorrem de maneira segura e ecoam na prática hospitalar como ferramenta que fortalece e consolida as teorias aprendidas no ambiente de sala de aula.

D Investigar as potencialidades e
Pesquisa qualitativa, por meio de observação participante e entrevista semiestruturada
Os resultados apontaram quatro principais potencialidades e desafios do ensino baseado em simulação para a formação médica: articulação entre teoria e prática; o erro como oportunidade de aprendizagem; relação entre mundo virtual e mundo real; fortalecimento do trabalho em equipe.

\section{Relato de}

Experiência atividade de simulação em diferentes cenários de emergências médicas na prática odontológica.

\section{A simulação de alta fidelidade realística para o ensino de emergências médicas na prática odontológica representa um cenário inovador e é eficaz para o processo de ensino e aprendizagem.}

F Apresentar e discutir ações e estratégias utilizadas por professores que ensinam o brinquedo terapêutico nos cursos de graduação em enfermagem.
Pesquisa
Ao refletir sobre as novas qualitativa, por meio de observação participante e entrevista semiestruturada

G Descrever o uso da simulação realística no ensino em um curso técnico em Enfermagem.
Análise descritiva A simulação contribuiu para o exploratória desenvolvimento de habilidades retrospectiva humanas e técnicas, que podem levar 
$\mathrm{H}$ Analisar as atitudes de alunos iniciantes do curso de graduação em Enfermagem diante do alcoolista
Estudo quanti qualitativo

o discente à descoberta de novas possibilidades de conhecimento, em conformidade com as demandas sociais, políticas, éticas e cidadãs.

I Comparar o efeito do emprego da estratégia de simulação combinada à teoria com a simulação, per se, no ganho de autoconfiança em profissionais de enfermagem durante o manejo do paciente em parada cardiopulmonar.

J Identificar se o emprego de simulação realística interfere na aquisição, retenção de conhecimento e na autoconfiança para administração de medicamento por via parenteral em estudantes de diferentes faixas etárias do curso de graduação em Enfermagem.

\section{Estudo No grupo experimental apesar da} experimental percepção relacionada à satisfação com abordagem ter se mostrado superior, não quantitativa houve diferença significativa. Mas a autoconfiança aumentou significativamente entre os profissionais do grupo experimental em relação ao controle.

$\begin{array}{cl}\text { Estudo } & \text { A maioria (85\%) dos estudantes } \\ \text { experimental } & \text { era do sexo feminino, com idade } \\ \text { quantitativo } & \text { média de } 24 \pm 5 \text { anos. Os estudantes } \\ & \text { apresentaram melhora significativa } \\ & \text { do conhecimento sobre a técnica } \\ & \text { de administração de medicamentos } \\ & \text { por via parenteral do pré-teste } \\ & \text { para o pós-teste após emprego da } \\ & \text { simulação realística. Os estudantes } \\ & \text { com idade }<29 \text { anos apresentaram } \\ & \text { melhora no desempenho na técnica } \\ & \text { de administração de medicamentos } \\ & \text { nas diferentes fases do estudo, } \\ & \text { comparados àqueles com idade } \geq 29 \\ & \text { anos. }\end{array}$




\section{Objetivos}

Métodos

\section{Resultados}

K Analisar o impacto da metodologia da simulação realística na visão dos acadêmicos de Enfermagem.

Estudo quanti- $\quad 98 \%$ dos acadêmicos afirmaram gostar qualitativo, a partir muito da aula com a metodologia de

da pesquisa- $\quad$ simulação; $97 \%$ relataram ter diferença ação na aprendizagem com a simulação; destes, $62 \%$ confirmaram ter maior rendimento no aprendizado quando utilizada a simulação com paciente simulado; e $35 \%$ mencionaram ter melhor rendimento quando utilizado o boneco estático.

L Relatar a experiência da utilização de

Relato de experiência iPads como ferramenta pedagógica e criativa em situações de simulação de aprendizagem no Laboratório de Cuidado Humano (LCH) do curso de Enfermagem.
Houve aumento de $50 \%$ na procura ao LCH para simulação de técnicas como recurso de aprendizado. Os alunos participantes relataram na avaliação institucional das disciplinas uma maior satisfação com o aprendizado de habilidades e raciocínio clínico.

\section{Pesquisa metodológica} e-Baby: integridade da pele junto a um painel de experts.

metodológica

O Serious game foi construído em

3D, com multimídia, incluindo animações e conteúdo embasado cientificamente. Validado pelos experts em todas as heurísticas, dentre os 36 itens analisados, $18(50 \%)$ foram considerados isentos de problemas, sendo que em nenhum dos itens houve mais que $25 \%$ de problemas classificados nos níveis 3 e 4, segundo a classificação de Nielsen.

$\mathrm{N}$ Identificar como os simuladores estão sendo usados para ensino, treinamento, terapia e exames na Medicina, com destaque para Oftalmologia.

\author{
Revisão \\ Perceberam-se as diferenças entre as \\ sistemática \\ áreas da Medicina, no que se refere \\ ao uso da realidade virtual. Apenas 10 \\ artigos explicitam com algum \\ aprofundamento os simuladores \\ oftalmológicos. A Oftalmologia é uma \\ das áreas que menos interagem com a
}


O Apresentar a modelagem conceitual de um simulador baseado em realidade virtual para treinamento de anestesia regional. realidade virtual, devido a dificuldades de montagem de imagens para serem aplicadas no simulador.
P Avaliar se há diferença no conhecimento teórico de alunos de enfermagem acerca do cateterismo vesical de demora entre alunos que aprenderam o procedimento pelo método tradicional de ensino e pelo método da simulação realística.
Relato de Experiência
Não existe um simulador totalmente virtual para o treinamento de técnicas de anestesia regional. $O$ processo de modelagem de um simulador para treinamento necessita da sistematização dos conceitos para guiar o processo de desenvolvimento.
Coorte histórico A média geral de acertos foi de 6,74, indicando conhecimento satisfatório. Não houve diferença no nível de conhecimento entre os grupos comparados $(\mathrm{p}=0,72)$.
Q Avaliar a progressão de competência de habilidades em endossuturas através de simulação realística em modelo de ensino metodizado.

\section{Estudo} prospectivo, analítico, observacional
Todos os alunos que participaram do curso absorveram os conceitos de videocirurgia. A maioria apresentou melhora constante e continuada, e no decorrer das etapas do treinamento, obtiveram progressão de habilidades adequadas, definindo competência e validação do modelo de ensino para atingir a proficiência.

R Avaliar o uso da aula simulada para

Estudo Verificou-se que $81 \%$ dos participantes o ensino de ações de enfermagem na intubação traqueal. quantitativo, quase possuíam conhecimento sobre experimental, tipo intubação traqueal e, após a estratégia pré e pós-teste de ensino, por meio de aula simulada, 98\% ampliaram seus conhecimentos 


\section{Objetivos}

Métodos

Estudo

experimental, exploratório e analítico participaram da simulação realística.

S Avaliar o conhecimento, a satisfação e autoconfiança de estudantes de cursos de graduação em Enfermagem e Medicina, que

$\mathrm{T}$ Comparar a opinião dos estudantes de graduação em enfermagem sobre a simulação realística e sobre o estágio curricular em cenário hospitalar.

Estudo $\quad$ No grupo experimental, $69,0 \%$

comparativo, concordaram totalmente que a prospectivo simulação consolidava o processo de ensino-aprendizagem. No grupo controle, $38,5 \%$ discordaram com o estágio em cenário hospitalar como estratégia isolada.
U Descrever experiência piloto de simulação in situ realizada em unidade de pronto atendimento, destacando oportunidades de avaliação de sistema de atendimento, trabalho em equipe e detecção de ameaças latentes à segurança.

V Descrever a construção de cenários e desenvolvimento da técnica de simulação realística em saúde sobre administração segura de medicamentos pela Enfermagem.

\section{Estudo A experiência proporcionou avaliação} prospectivo, técnica, comportamental e sistemas. observacional Detectou quatro ameaças latentes à segurança que permitiu reflexão guiada sobre trabalho em equipe. 


\section{Objetivos}

W Descrever o método do exame

clínico objetivamente estruturado no ensino de suporte básico de vida na graduação de Enfermagem.
Métodos

Relato de

Experiência

\section{Resultados}

$\mathrm{X}$
Analisar o referencial teórico acerca da simulação como estratégia de ensino para o curso de Enfermagem.
Revisão Integrativa A maior parte dos estudos adotou uma abordagem descritiva e prospectiva. Foram citados como benefícios a construção de competências e o pensamento crítico, assim como o desenvolvimento de uma participação ativa do estudante; aumento da confiança dos estudantes na comunicação com os pacientes, remoção da ansiedade, possibilidade de treinamento para situações de emergências e facilitação no gerenciamento de evento de crise e ampliação da aprendizagem contextual. A presença de limitações para o realismo dos simuladores de alta fidelidade, bem como do aumento da carga de trabalho, superestimação da confiança sobre o desempenho real e limitação na disponibilidade de simuladores foram citados como desvantagens nos artigos.

\section{Refletir sobre as perspectivas}

Y atuais de ensino e aprendizagem no contexto da formação em saúde e Enfermagem a partir do uso da simulação enquanto metodologia ativa.

\section{Estudo teórico reflexivo}

\section{A simulação é uma estratégia de ensino que permite que as pessoas experimentem a representação de um evento real com o propósito de praticar, aprender, avaliar ou entender estas situações. Nas experiências}




\section{Objetivos}

Métodos

Resultados

em situações reais e simuladas, os resultados pontuam a ideia de ressignificação da aprendizagem e dos mecanismos de articulação e construção de novos saberes, e contribuem para uma formação mais próxima das necessidades exigidas pela sociedade atual.

Os resultados foram divididos em categorias para facilitar a compreensão de como a simulação realística está sendo usada no ensino nas escolas de saúde. Dessa forma, as categorias foram: simulação realística como ferramenta de aprendizado nas graduações em saúde, emprego da técnica na segurança do profissional e do paciente, limitações da metodologia e desafios da simulação realística na saúde.

\section{DISCUSSÃo}

Analisando as publicações estudadas identificaramse diversos achados relacionados às contribuições da simulação realística nas graduações em saúde, tornando-se possível entrever as potencialidades, fragilidades e desafios da técnica.

\section{Simulação realística como ferramenta de aprendizado nas graduações em saúde}

Com o intuito de compreender como os estudantes de saúde se posicionam diante da utilização da simulação realística, três estudos mostraram que essa técnica permite que os graduandos tenham uma vivência prévia da profissão e consigam relacionar a teoria vista na sala de aula com a prática, auxiliando na retenção dos conteúdos. O aprimoramento de habilidades e atitudes como liderança, tomada de decisão e trabalho em equipe mostrou-se como benefício assegurado pela simulação $0^{6,10}$. Outros autores também concluíram que a simulação ajuda o estudante a visualizar, questionar e validar informações antes de vivenciar a prática ${ }^{11,12}$.

Competências como relação profissional da saúde/ paciente também mostraram ser fatores que podem ser aperfeiçoados com a simulação realística. Um estudo realizado com atores simulados mostrou clara melhora de habilidades de comunicação nos âmbitos comportamental, conceitual e emocional ${ }^{13}$.

A autoconfiança foi tema de estudo em 3 artigos selecionados. Em um estudo foi realizada escala de satisfação e autoconfiança em que os estudantes demonstraram benefícios significativos com a simulação realística ${ }^{14}$. Outras publicações mostraram que a integração do método tradicional com simulação, teoria e prática mostrou-se eficaz para a aquisição e, consequentemente, evolução do conhecimento ${ }^{15,16}$.

Com a mudança do método tradicional de ensino para as metodologias ativas, a simulação mostra-se mais atraente para os alunos. Os estudos de Rohrs17 e Figueiredo $^{18}$ mostraram maior satisfação dos estudantes com o uso da técnica em comparação 
com o método tradicional isolado. Rohrs estudou a opinião dos alunos participantes de uma simulação realística que se mostraram mais satisfeitos com as aulas que utilizam a ferramenta de ensino. Já Figueiredo mostrou que a procura pelo laboratório de cuidados humanos da faculdade em estudo aumentou 50\% depois de incluídas novas tecnologias no laboratório, o que permitiu melhor satisfação com o aprendizado de habilidades e raciocínio clínico.

O uso de novas tecnologias também contribui para a melhor prática da simulação realística, embora não se restrinja a elas. Simulações com realidade virtual foram empregadas em estudos que utilizaram jogos sobre cuidados com a pele em neonatos prematuros no ensino da Enfermagem ${ }^{19}$. Também são utilizadas para o aprendizado de Otoscopia, Oftalmologia, Cirurgia e Anestesiologia com a utilização de pacientes virtuais que são modelos de representação de humanos virtuais ${ }^{20,21}$.

A simulação realística mostra-se essencial, também em habilidades específicas como na realização de procedimentos. Lopes ${ }^{22}$, Moura-Júnior ${ }^{23}$ e Alves $^{24}$ apresentaram estudos com simulação realística empregada em procedimentos de cateterismo vesical, realização de endosuturas por via laparoscópica e intubação respectivamente. Embora o primeiro estudo não tenha mostrado diferença significativa entre o grupo controle e o grupo que realizou simulação realística, depois da utilização da simulação houve melhora do conhecimento sobre o tema. No estudo sobre endosuturas, os alunos que participaram do curso absorveram os conceitos de videocirurgia e a maioria apresentou melhora da habilidade. Dessa forma, a simulação mostrouse válida como modelo de ensino para atingir a proficiência da técnica. O estudo sobre intubação também mostrou benefícios da metodologia que permitiu que $98 \%$ ampliassem seus conhecimentos sobre o assunto.

No estudo de Ferreira, Guedes e Oliveira ${ }^{25}$, a média obtida após simulação foi superior à média atingida pelos sujeitos submetidos ao método tradicional, demonstrando ser a simulação uma grande ferramenta para a aquisição de conhecimento. O estudo de Valadares e Magro ${ }^{26}$ conseguiu evidenciar que os estudantes preferem as metodologias ativas, e quando não é possível aplicá-las totalmente, deve ser associada ao método tradicional.

\section{Simulação realística empregada na segurança do profissional e do paciente}

A simulação realística foi descrita como um ambiente seguro para os pacientes nos estudos ${ }^{7,23}$. A segurança do paciente é um fator decisivo na implementação da simulação realística nas instituições de ensino mundiais, além de ser efetiva nas necessidades práticas dos estudantes em adquirir habilidades essenciais de forma que se consiga a proficiência, promovendo o bem-estar dos pacientes ${ }^{7}$.

Além de ser seguro e ético, por não utilizar pacientes reais para o aprendizado, a simulação realística também pode promover cenários para simulação de práticas que detectem problemas na segurança do paciente. Um artigo apresentou uma experiência de uma simulação in situ realizada em unidade de pronto atendimento, destacando oportunidades de avaliação do sistema de atendimento, trabalho em equipe e detecção de ameaças latentes à segurança. Nesse estudo, detectou-se quatro ameaças latentes à segurança que permitiu uma reflexão de toda a equipe do serviço, o que possibilitou o aperfeiçoamento de práticas em segurança do paciente ${ }^{14}$. A simulação in situ é uma técnica, em que a simulação é realizada dentro do próprio equipamento de saúde, o que permite melhor fidedignidade do cenário de prática, sem ter o inconveniente de usar pacientes reais para o aprendizado ${ }^{27}$.

Um artigo relatou uma experiência sobre a administração segura de medicamentos, em que foram construídos quatro cenários baseados em eventos adversos ocorridos na instituição hospitalar. 
Houve preocupação com a fidelidade dos cenários e foi realizada a execução do debriefing ${ }^{28}$. Nessa etapa foi explanado sobre a tomada de decisão e as estratégias de prevenção de eventos adversos futuros. A importância do debriefing se dá em razão da síntese e sumarização dos principais pontos que deveriam acontecer na simulação, sem o julgamento dos participantes da simulação, permitindo a integração e confiança tanto em competências técnicas quanto interpessoais ${ }^{29}$.

\section{Limitações da simulação}

As principais desvantagens da simulação realística é o desafio singular para os estudantes iniciantes. Há limitações para o realismo dos simuladores de alta fidelidade, bem como do aumento da carga de trabalho, do custo para estruturação de um cenário realístico, superestimação da confiança sobre o desempenho real e limitação na disponibilidade de simuladores ${ }^{30}$. No entanto, cabe ressaltar que a simulação realística não necessariamente exige simuladores de alta fidelidade para ser realizada. Cenários podem ser montados com equipamentos facilmente encontrados nas escolas de saúde ou através de atores simulados, que podem ser os próprios alunos da instituição.

A simulação esbarra, também, na necessidade de engajamento do corpo docente para a realização e implementação dessa metodologia, de modo que ela contribua no processo de ensino-aprendizagem ${ }^{30}$.

\section{Desafios da simulação realística na saúde}

Dos estudos analisados, 19 são das graduações em enfermagem, 7 envolvem alunos da graduação em medicina e apenas 3 envolvem equipes interdisciplinares. A maior parte das publicações mostra a simulação realística empregada em cenários de urgência e emergência, centro cirúrgico, enfermarias e realização de procedimentos.
Poucos estudos utilizam a técnica, em situações de comunicação ou atenção primária, que poderiam facilmente ser aplicadas nas graduações de ensino em saúde.

A simulação consegue abranger os amplos espectros das mais diversas áreas da saúde, e ainda consegue possibilitar a inter-relação entre elas, capacitando os profissionais para ações integrais em saúde ${ }^{25}$. Dessa forma, em relação aos desafios inerentes ao uso da simulação realística, há a necessidade de maior engajamento das instituições de ensino, principalmente de outras áreas além da Enfermagem e Medicina, como Biomedicina, Farmácia, Terapia Ocupacional e psicologia, para a implementação dessa técnica, já consagrada pelas instituições de ensino mundiais. Além disso, muito pouco se produz, em relação às simulações realísticas interdisciplinares. Essa técnica permite a criação de experiências de aprendizagem realistas em que os diversos papéis, responsabilidades e a identidade profissional possam ser aprendidas, desenvolvidas e avaliadas, além de ser essencial para a redução dos estereótipos negativos nas relações interprofissionais.

Os estudos também mostram que a simulação é uma metodologia ativa que desenvolve um formando melhor tecnicamente, ético e humano. Nas escolas de saúde que se preocupam com a qualidade do ensino são necessários investimentos no ensino prático simulado, em contexto laboratorial, de elevada qualidade, com embasamento científico, ético e respaldo legal. Dessa maneira a graduação, utilizando a simulação, tem o dever de ser exemplo de boas práticas, começando pelo respeito integral pela pessoa humana ${ }^{31}$.

\section{CONCLUSÕES}

A análise das publicações sobre simulações realísticas nas escolas de saúde brasileiras, nos últimos dez anos, mostrou escassez de pesquisas sobre o tema 
nas graduações além da enfermagem e medicina. Áreas como emergência e cirurgia são priorizadas e há poucos estudos em cenários de prática como a atenção primária.

Os estudos com simulação realística interprofissional também são escassos, mas deveriam ser prioridade nas escolas de saúde e na assistência. É fato que, depois da graduação, a assistência à saúde ocorra de forma interdisciplinar. Assim, é essencial que, desde a graduação, os acadêmicos de diferentes áreas de ensino tenham contato, para o aperfeiçoamento de competências necessárias.

A elaboração dessa revisão integrativa sobre o uso da simulação realística, enquanto técnica de transformação do processo ensino-aprendizagem e da assistência em saúde, mostrou seus impactos positivos na formação e qualificação dos graduandos dos cursos em saúde e profissionais da assistência. A demanda por profissionais mais qualificados tecnicamente, e que trabalhem baseados em evidências científicas de forma ética e humanizada, aumenta cada vez mais e consolida a simulação realística como uma técnica válida e essencial para a formação dos profissionais de saúde. É uma metodologia ativa que faz do aluno, o protagonista de seu aprendizado e promove o processo de ensinoaprendizagem de forma ética, porque não utiliza pacientes reais como ferramenta de ensino, como ocorria em tempos passados.

Também foi possível identificar a existência de limitações da simulação realística e fragilidades na sua implementação, como custos para estruturação dos cenários e necessidade de engajamento das instituições de ensino. Esses são verdadeiros desafios a serem enfrentados para que o ensino nas graduações em saúde alcance uma qualidade cada vez maior.

\section{REFERÊNCIAS}

1. Issenberg SB, Pringle S, Harden RM, Khogali S, Gordon MS. Adoption and integration of simulation-based learning technologies into the curriculum of a UK Undergraduate Education Programme. Med Educ. 2003;37(s1):42-9.

2. Jones F, Passos-Neto CE, Braghiroli OFM. Simulation in Medical Education: Brief history and methodology. Princ Pract Clin Res. 2015;2(1):56-63.

3. Okuda Y, Bryson EO, DeMaria S, Jacobson L, Quinones J, Shen B, i in. The Utility of Simulation in Medical Education: What Is the Evidence? Mt Sinai J Med A J Transl Pers Med [Internet]. 2009;76(4):330-43. Disponível em: http://doi.wiley.com/10.1002/msj.20127

4. So HY, Chen PP, Wong GKC, Chan TTN. Simulation in medical education. $\mathrm{J} R$ Coll Physicians Edinb [Internet]. 2019;49(1):52-7. Disponível em: http://doi.wiley. com/10.1002/9781118472361.ch13

5. Mendes KDS, Silveira RCCP, Galvão CM. Revisão integrativa: método de pesquisa para a incorporação de evidências na saúde e na enfermagem. Texto Context Enferm. 2009;17(4):758-64.

6. Costa RRO, Medeiros SM, Martins JCA, Cossi MS, Araújo MS. Percepção de estudantes da graduação em enfermagem sobre a simulação realística. Rev Cuid. 2017;8(3):1799.

7. Brandão CFS, Collares CF, Marin HF. A simulação realística como ferramenta educacional para estudantes de medicina. Sci Med (Porto Alegre). 2014;24(2):187-92.

8. Magro MCS, Silva TS, Moreira SCR, Silva KGN, Barreto DG, Santos CE, i in. Vivência prática de simulação realística no cuidado ao paciente crítico: relato de experiência TT - Practical experience of realistic simulation in the care of critically ill patients: experience report. Rev baiana enferm [Internet]. 2012;26(2):556-61. Disponível em: https://portalseer.ufba.br/index.php/enfermagem/article/ view/6544/6360\%0Ahttp://www.portalseer.ufba.br/index. php/enfermagem/article/view/6544/6360

9. Dourado ASS, Giannella TR. Ensino Baseado em Simulação na Formação Continuada de médicos: análise das Percepções de alunos e Professores de um Hospital do Rio de Janeiro. Rev Bras Educ Med. 2014;38(4):460-9. 
10. Motta RHL, Ramacciato JC, Oliveira LB, Camargo MA, Pedro RN, Martins RS, Barros RF. i in. Simulação de alta fidelidade realística para o ensino de emergências médicas na prática odontológica: relato de experiência. Rev da ABENO. 2018;18(2):174-81.

11. Maia EBS, Ohara CVS, Ribeiro CA. Ensino do brinquedo terapêutico na graduação em enfermagem: ações e estratégias didáticas. Texto Context - Enferm [Internet]. 2019;28:1-17. Disponível em: http://www.scielo.br/scielo. php?script=sci_arttext\&pid=S0104-07072019000100301\&ln $\mathrm{g}=\mathrm{en} \& \mathrm{t} \operatorname{lng}=\mathrm{en}$

12. Sanino GEC. O uso da simulação em enfermagem no Curso Técnico de Enfermagem. J Heal Inform. 2012;4(Número Especial):148-51.

13. Rodrigues SB, Souza J. Atitudes de estudantes iniciantes da graduação em enfermagem diante do paciente alcoolista. SMAD, Rev Eletrônica Saúde Ment Álcool Drog. 2018;14(1):37-44.

14. Kaneko RMU, Couto TB, Michael II, Coelho MM, i in. Simulação in Situ, uma Metodologia de Treinamento Multidisciplinar para Identificar Oportunidades de Melhoria na Segurança do Paciente em uma Unidade de Alto Risco In Situ Simulation, a Multidisciplinary Training Method to Identify Opportunities to Improv. Rev Bras Educ Med [Internet]. 2015;39(2):286-93. Disponível em: http://dx.doi.org/10.1590/1981-52712015v39n2e00242014

15. Mesquita HCT, Santana BS, Magro MCS. Effect of realistic simulation combined to theory on self-confidence and satisfaction of nursing professionals. Esc Anna Nery. 2019;23(1):1-6.

16. Nascimento MS, Magro MCS. Realistic simulation: method of improving knowledge and self-confidence of nursing students in the administration of medication. Reme Rev Min Enferm. 2018;22:1-5.

17. Rohrs RMS, Santos CF, Barbosa RS, Schulz RDS, Carvalho MB. Impacto da metodologia de simulação realística na graduação de enfermagem. Rev Enferm UFPE line. 2017;11(12):5269.

18. Figueiredo AE. Laboratório de enfermagem: estratégias criativas de simulações como procedimento pedagógico. Rev Enferm da UFSM. 2015;4(4):844-9.

19. Aredes NDA, Dias DMV, Fonseca LMM, Campbell SH, Martins JCA, Rodrigues MA. E-baby skin integrity: evidence-based technology innovation for teaching in neonatal nursing. Esc Anna Nery. 2018;22(3):1-9.

20. Carvalho JA. Oftalmologia e realidade virtual. Rev Bras Oftalmol. 2012;71(1):40-7.

21. Costa T, Gomes DC, Machado LS, Azevedo WS. Modelagem conceitual de simulador virtual para treinamento em anestesia regional. J Heal Informatics. 2016;8(1):247-54.

22. Lopes AM, Souza CC, Teixeira AO, Salgado PO, Prado Júnior PP. Conhecimento teórico de estudantes de enfermagem sobre o cateterismo vesical de demora. Rev Enferm do Centro-Oeste Min. 2018;8:1-8.

23. Moura-Júnior LG de, Ramos A, Campos JM, Ferraz AA, Rocha HAL, Costa GO. Teaching model for evaluation of the ability and competence progress in endosuture in surgical skill laboratory. Arq Bras Cir Dig (São Paulo) [Internet]. 2017;30(4):256-9. Disponível em: http://www. scielo.br/scielo.php?script=sci_arttext\&pid=S010267202017000400256\&lng=en\&tlng=en

24. Alves MG, Morais CCP, Oliveira JM, Silva AT, Pereira VOS, Dalri MCB. Aula Simulada no Ensino de Ações de Enfermagem na Intubação. J Nurs UFPE line - ISSN 19818963 [Internet]. 2018;12(3):592-8. Disponível em: https:// periodicos.ufpe.br/revistas/revistaenfermagem/article/ view/230940/28001

25. Ferreira RPN, Guedes HM, Oliveira DWD, Miranda JL. Simulação realística como método de ensino no aprendizado de estudantes da área da saúde. Rev Enferm do Centro-Oeste Min. 2018;8(5):7877-86.

26. Valadares AFM, Magro MCS. Opinion of nursing students on realistic simulation and the curriculum internship in hospital setting Opinião dos estudantes de enfermagem sobre a simulação realística e o estágio curricular em cenário hospitalar. Acta Paul Enferm [Internet]. 2014;27(2):138-81. Disponível em: http://dx.doi. org/10.1590/1982-

27. Kurup V, Matei V, Ray J. Role of in-situ simulation for training in healthcare. Curr Opin Anaesthesiol. 2017;30(6):755-60.

28. Cogo ALP, Lopes EFS, Perdomini FRI, Flores GE, Santos MRR. Construção e desenvolvimento de cenários de simulação realística sobre a administração segura de medicamentos. Rev Gaúcha Enferm. 2019;40(spe). 
29. Oliveira DAL, Silva JCB. Exame clínico objetivamente estruturado no ensino de suporte básico de vida. Rev Enferm UFPE line. 2018;12(4):1185-90.

30. Barreto DG, Silva KGN, Moreira SSCR, Silva TS, Magro MCS. Simulação Realística como estratégia de ensino para o curso de graduação em Enfermagem: Revisão Integrativa. Rev Baiana Enferm. 2014;28(2):208-14.

31. Costa RRO, Medeiros SM, Martins JCA, Menezes RMP, Araújo MS. O uso da simulação no contexto da educação e formação em saúde e enfermagem: uma reflexão acadêmica. Espaço para a Saúde - Rev Saúde Pública do Paraná. 2017;16(1):59. 